\title{
ASO Author Reflections: Changes in Breast Cancer Care and Patient Perception During the Initial Wave of COVID-19
}

\author{
Kaitlyn Kennard, $\mathrm{MD}^{1,2,3}$ (D) , Lina M. Sizer, $\mathrm{DO}^{3}$, and Thomas G. Frazier, $\mathrm{MD}^{3}$ \\ ${ }^{1}$ Lankenau Institute for Medical Research, Wynnewood, PA; ${ }^{2}$ Lankenau Medical Center, Wynnewood, PA; ${ }^{3}$ The Bryn \\ Mawr Hospital, Bryn Mawr, PA
}

\section{PAST}

The coronavirus disease 2019 (COVID-19) virus was categorized as a pandemic in March 2020. At this time, restrictions were instituted based on state case counts, to decrease both transmission and the burden on health systems. By 19 March 2020 'elective procedures' in Pennsylvania were prohibited. In response, the Society of Surgical Oncology and the COVID-19 Pandemic Breast Cancer Consortium developed recommendations on how to triage breast cancer patients. ${ }^{1,2}$

The aim of this study was to capture the changes made in the multidisciplinary care of breast cancer patients in a community hospital during the initial phases of the COVID-19 pandemic. We also sought to assess if these changes were associated with negative reactions from patients related to their overall mental health, breast cancer care, and outlook. ${ }^{3}$

\section{PRESENT}

Our study showed that $44 \%$ of breast cancer patients in a community hospital experienced a change (CTX) in their breast cancer care during the initial wave of COVID-19. The changes seen were either a delay in surgical date alone or a delay in surgical date with the use of neoadjuvant endocrine therapy (NET). Time to surgery was only 24 days for the no change (NC) cohort but 82 days for CTX.

(C) Society of Surgical Oncology 2021

First Received: 20 December 2020

Accepted: 24 December 2020;

Published Online: 28 January 2021

K. Kennard, MD

e-mail: kaitlyn.kennard@gmail.com
The median duration of NET was 78 days. The Generalized Anxiety Disorder 2-item (GAD-2) questionnaire, which assesses levels of anxiety and depression, was positive for about $30 \%$ of patients in both the CTX and NC cohorts, with no significant difference. Despite similar GAD-2 scores, over half of the CTX cohort believed COVID-19 affected their treatment outlook, while only one-quarter of the NC cohort reported this.

\section{FUTURE}

A cancer diagnosis is a very stressful time in an individual's life; amidst a pandemic, this presents a new set of challenges. We anticipated an increase in anxiety and depression for the CTX cohort, however this cohort had similar GAD-2 scores as both NC and historical pre-pandemic breast cancer patients. ${ }^{4}$ As we are currently facing a second wave of the pandemic, delays in elective surgical procedures are again being initiated based on a state's capacity. From this study, we anticipate that women with informed consent can tolerate delays in surgical intervention without a significant increase in levels of perceived anxiety and depression. We recommend careful consideration of delays in intervention based on national recommendations and local COVID-19 case volumes; ${ }^{5}$ however, if delays are necessary, we anticipate women can move through their treatment without additional psychological distress.

\section{REFERENCES}

1. Dietz JR, Moran MS, Isakoff SJ, et al. Recommendations for prioritization, treatment, and triage of breast cancer patients during the COVID-19 pandemic. the COVID-19 pandemic breast cancer consortium. Breast Cancer Res Treat. 2020;181(3):487-97.

2. Society of Surgical Oncology. Resource for management options of breast cancer during COVID-19. 2020. https://www.surgonc. 
org/wp-content/uploads/2020/03/Breast-Resource-during-COVID19-3.23.20.pdf.

3. Kennard K, Williams AD, Goldblatt LG, et al. COVID-19 pandemic: changes in care for a community academic breast center and patient perception of those changes. Ann Surg Oncol. 2021. https://doi.org/10.1245/s10434-020-09583-3.

4. Tsaras K, Papathanasiou IV, Mitsi D, et al. Assessment of depression and anxiety in breast cancer patients: prevalence and associated factors. Asian Pac J Cancer Prev. 2018;19(6):1661-9.
5. COVID-19: guidance for triage of non-emergent surgical procedures. https://www.facs.org/covid-19/clinical-guidance/triage. Accessed 24 July 2020.

Publisher's Note Springer Nature remains neutral with regard to jurisdictional claims in published maps and institutional affiliations. 